\title{
Exploring the threats in Cell Phone as a Portable Storage Device
}

\author{
Vaishali Mishra, \\ Lecturer, Vishwakarma Institute of \\ Information Technology, Pune
}

\author{
Ashutosh Mishra, \\ Graduate Student, University of \\ Missouri, Columbia
}

\begin{abstract}
The introduction of personal computing and wireless communication technology provides an option for on site device software updating and data retrieving. This is especially true for any devices sitting in a remote site where computing network is not accessible. In many advanced computing systems, frequent software updating and configuration profiles refreshing are required. This is clumsy and error prone procedures when users are not familiar with the operating systems. Suppose all the necessary files and programs are predefined in a mobile phone. All necessary files and software can be transferred to the corresponding computing devices and PCs at remote sites through wireless communication links such as Bluetooth/infrared. This idea helps to solve the initial installation cost of a communication network to a remote site.
\end{abstract}

The full text of the article is not available in the cache. Kindly refer the IJCA digital library at www.ijcaonline.org for the complete article. In case, you face problems while downloading the full-text, please send a mail to editor at editor@ijcaonline.org 